\title{
WiFi: Internet Connection Sharing over WI-FI
}

\author{
Javed Ahmad Shaheen \\ Computer Science Department, GC University Faisalabad, Pakistan \\ javedmatyana@yahoo.com
}

\begin{abstract}
With variety of existing internet connections like dial-up, ISDN, lease line there are different ways to share the connection among internet users. But the question arises how efficient your sharing technique is with respect to speed and security. With the advent of wireless technologies, sharing of internet connection (lease line) can be made efficient. If you want to design a Wi-Fi network for a complex, then how to go for it? As computer and the Internet have permeated everyday life, the Internet communication has been a common activity of Internet user. It provides convenient channels to increase social contacts with others. Online interaction facilitated by digital connection creates a situation in which users negotiate their time online with real-life socializing; this paper throws some ideas on the design of Wi-Fi network. In this paper, we mainly address the problem of sharing wireless internet bandwidth among the buildings (also the users within the building) under one complex and some ways of setting up wired/wireless network based on the requirement basic components. Different network layouts are also presented here. Installation of the given layouts has been practically tested and the results are satisfying and security also is achieved.
\end{abstract}

Keywords: Wi-Fi, LAN, VLAN

\section{Introduction}

With the upcoming development of wireless technologies, setting up a Wi-Fi network is becoming popular day by day. But the design of the Wi-Fi network and choosing the configuration, expaertise help is required. Once you are aware of all the facts related to the network then setting up a new network would not be a problem. Suppose you want to share internet bandwidth received from ISP provider among number of buildings within a complex, a combination of wired and wireless network can also be set up. There are two types of wireless networks (Indoor \& Outdoor). A Wireless network basically utilizes the wireless frequencies $2.4 \mathrm{Ghz}$ or $5 \mathrm{Ghz}$ spectrum to transmit the data, voice and video from one point to another using the wireless signals as the medium but it is very rare to have a total stand-alone wireless network as the wireless transmission and reception is restricted from the client (PC/Laptop/Wi-Fi Phone etc) to the Wireless Access Point. Beyond that, the data is carried in the wired backbone network. Look at the below Wi-Fi architecture diagram 1 for clarification.

\subsection{Indoor RF Wireless Networks}

Several factors are to be considered while dealing with installation and expansion of indoor wireless networks. Manufacturers indicate a typical range of their wireless access points and routers. Usually these range estimates permission to make digital or hard copies of all or part of this work for personal or classroom $m$ use is granted without fee provided that copies are not made or distributed for profit or commercial advantage and that copies bear this notice and the full citation on the first page. To copy otherwise, or

Received (July 15, 2017), Review Result (October 19, 2017), Accepted (October 26, 2017) 
republish, to post on servers or to redistribute to lists, requires prior specific per mission and/or a fee and generally, there will be many obstacles present in an indoor installation which would affect performance drastically. Signals will not penetrate metal or concrete walls. Factors that will reduce range and affect coverage area include metal studs in walls, concrete fiberboard walls, aluminum sliding, and foil-backed insulation in the walls, pipes and electrical wiring. Also any wireless equipment, cordless phones, radio transmitters and electrical equipment will affect performance to determine the success of an installation, reflections (when wireless signals "bounce" off objects) and multipath (when wireless signals travel in multiple paths arriving at the receiver at different times) should be considered as important as signal strength.

\subsection{Outdoor RF Wireless Networks}

Like Indoor networks, Outdoor RF wireless networks experience reflections and multipath. Optimal line of sight is accepted for outdoor installations. Generally, trees and leaves are obstructions to 802.11 frequencies and they partially or entirely block the signal if not cleared. A site survey has to be done before deploying any wireless network. The survey entails installing an access point at each location where user groups are located and then monitoring the wireless signal strength. This will help you in assessing the number of access points required to provide sufficient coverage and in which location you require an access point for optimal wireless connectivity.

\subsubsection{WLAN Architecture and Working}

The process of bringing together the parts of computer hardware in computer networking is called as computer architecture so if we use this architectural technique in Wireless LAN or WiFi is called as Wireless LAN Architecture. It is a technique of designing and arrangement of different components in Wireless local area networking (WLAN) device in a specific way. Special type of device which is the combination of transmitter and receiver called as transceiver which is an essential part for standard Wireless LAN architecture. It is known as Access points. Wireless LAN architecture is composed of different components in establishing the local area network between different operating systems; these are Access Point, Bridge, and client.

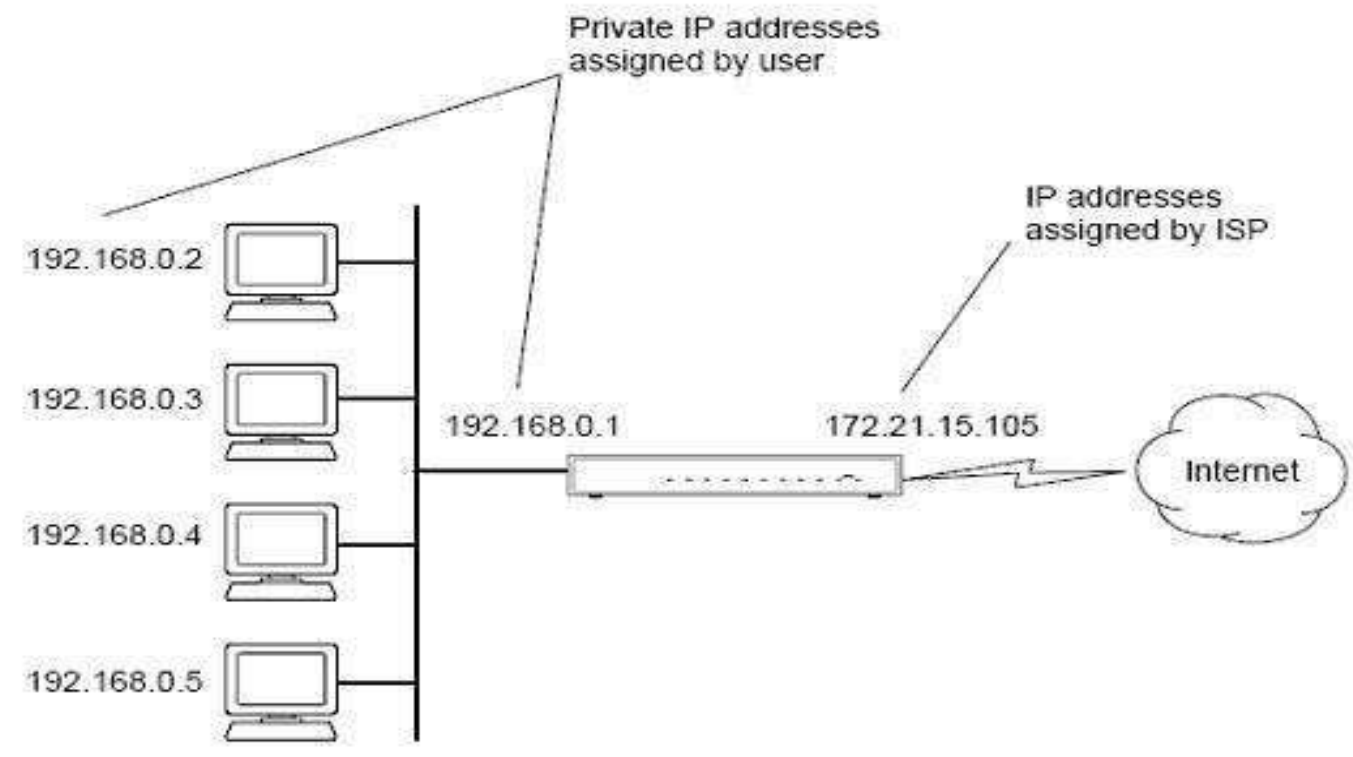

Figure 1. WLAN with Access Point 


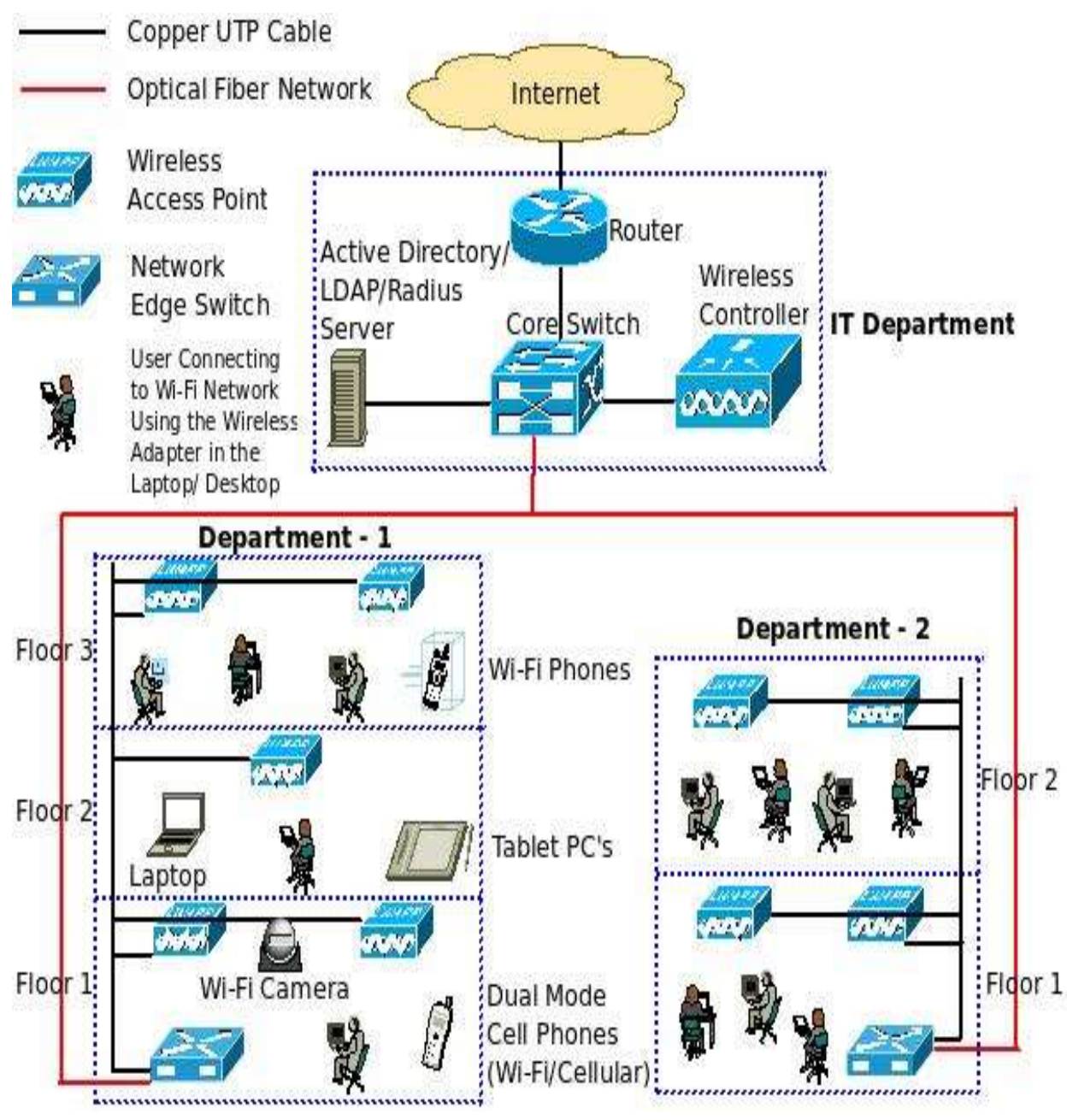

Figure 2. WLAN

\subsection{Some Basic Components Required to Setup Wi-Fi Network}

Point to Point

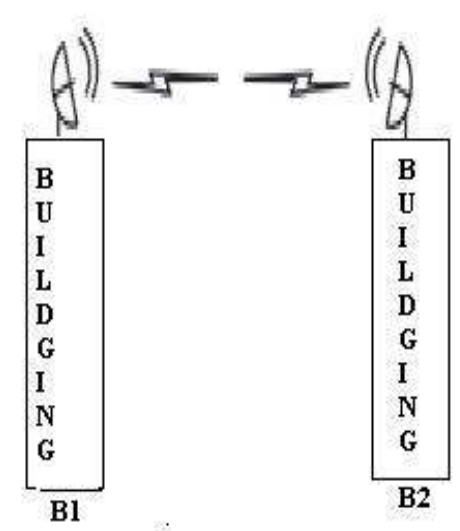

Point to Multipoint

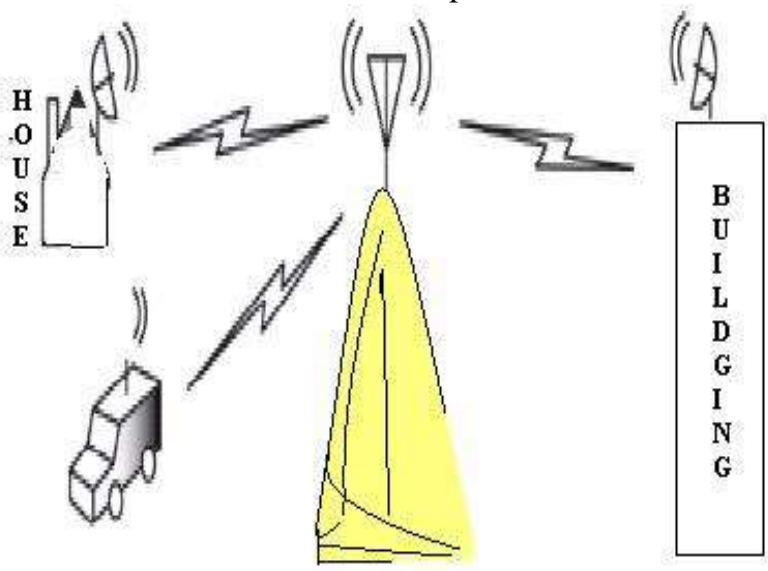

TOWER ANTENNA

Figure 3. WLAN Architectures 


\subsubsection{Antenna}

It is part of a radio communications system intended to radiate and/or collect radio frequency energy. Different types of antenna available, these are:

\subsubsection{Omni-Directional Antenna}

An antenna that radiates or receives RF energy in a 360 degree patterns about an axis.

\subsubsection{Motorola Canopy}

Motorola Canopy doesn't require access point. It has high-speed Internet access, web browsing, e-mail and supports advanced applications like VoIP and multimedia services.

\subsubsection{Yagi-uda}

A narrow band directional antenna named after one of its inventors, which consists of a beam supporting a series of metallic elements or rods.

\subsubsection{Access Points}

Is a device that allows wireless communication devices to connect to a wireless network using Wi-Fi.

\subsubsection{Power over Ethernet}

POE is also called Active Ethernet, it eliminates the need to run 110/220 VAC power to Wireless Access Points and other devices on a wired LAN. Using Power-overEthernet system installers need to run a single CAT5 Ethernet cable that carries both power and data to each device. This allows greater flexibility in locating the AP's and network devices and significantly decreasing installation costs in many cases.

\subsubsection{Distribution Cables}

CAT5/CAT6/CAT7 can be used.

\subsubsection{Switches}

Two switches are used in this mechanism.

Zyxel switch: It's a Manageable switch.

Cisco Switch: - It's a Manageable switch. VLAN is enabled by default

\subsection{LANS and VLANS}

\subsubsection{LAN Segmentation}

In LAN segmentation all the segments will be in the same broadcast domain. This effectively, reduces the efficiency of the network. LAN segmentation is shown in Figure 2. 


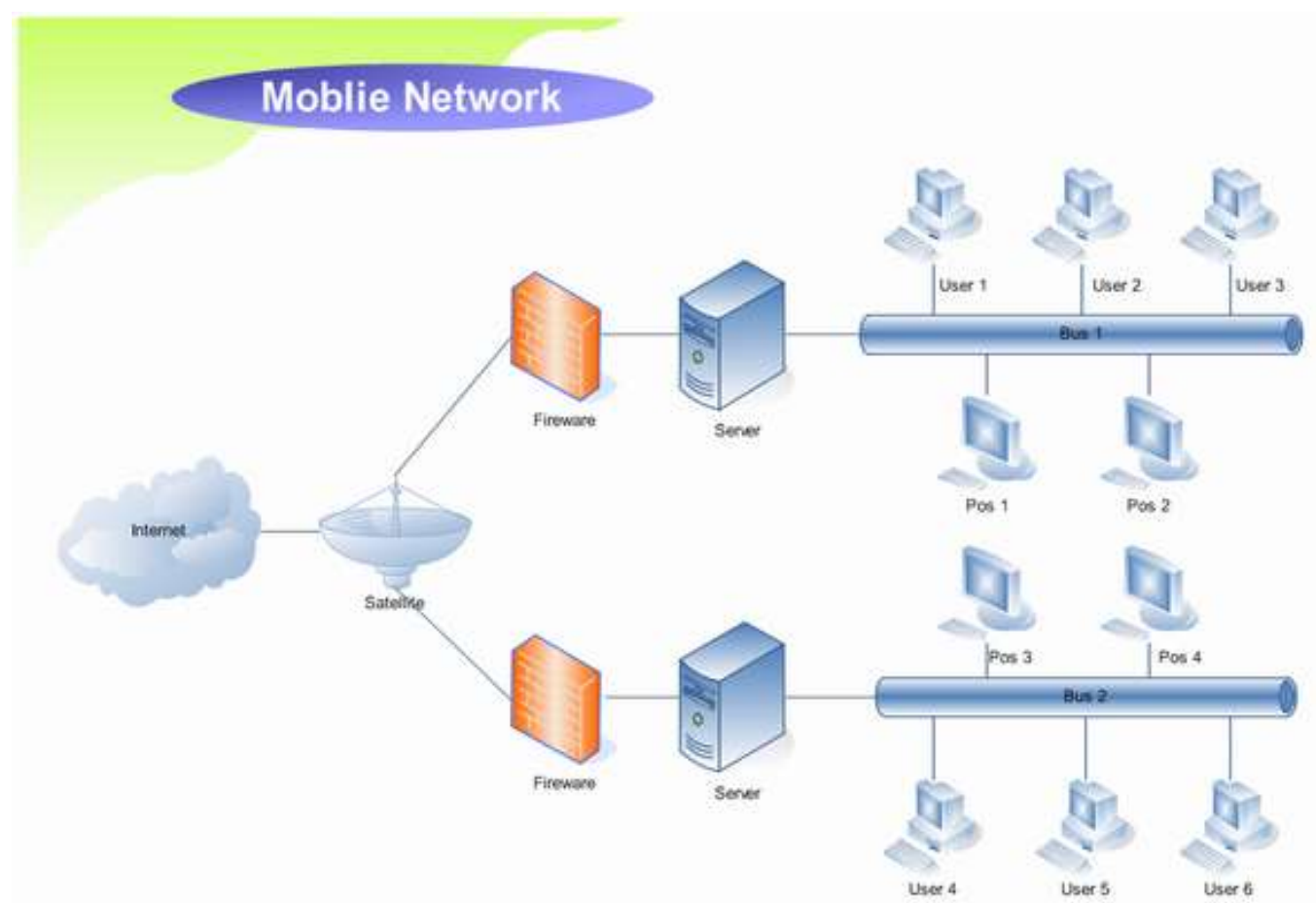

Figure 3. LAN Segmentation

\subsubsection{VLANs}

In VLANs the broadcast domain gets divided into the number of VLANs. If there are three VLANs, as shown in the Figure 3, the broadcast domain will be split into three. A router is required to route traffic between VLANs, and each VLAN is in different broadcast domain.

\subsubsection{VLAN Types}

- VLAN membership by Port

- VLAN membership by MAC

- Address Membership by Protocol Type

- Membership by IP Subnet Address

\subsubsection{VLAN Membership by Port}

Which port of a Switch belongs to which VLAN is defined in this section. Now, any workstation connected to a particular port will be automatically assigned that VLAN. For example, in a Switch with eight ports, ports 1-4 may be configured with VLAN1, and ports 5-8 may be configured with VLAN2.Main disadvantage of this method is that whenever a user moves from one place to another with associated workstation, Switch port reconfiguration has to be done.

\subsubsection{VLAN Membership by MAC address}

In this method, membership in a VLAN is based on the MAC address of the user workstation.MAC address is used by a Switch (that participates in VLAN) to assign a VLAN to each workstation .Now, whenever a workstation moves from one place to another, the corresponding Switch will automatically discover the associated VLAN 
based on the MAC address of the workstation. Since the MAC address is normally inseparable from that of the workstation, this method of VLAN membership is more amenable to workstation moves.

\subsection{Communication between VLANs}

Different protocols are there for communicating between VLANs. They are also known as VLAN trunking protocols. These are:

1. Inter-Switch Link Protocol (ISL)

2. IEEE 802.10 Protocol

3. IEEE 802.1Q Protocol

4. ATM LANE Protocol

\subsection{ATM LANE Fast Simple Server Replication Protocol (FSSRP)}

Inter-Switch Link Protocol (ISL): For interconnecting two VLAN-capable Ethernet, Fast Ethernet, or Gigabit Ethernet devices ISL protocol is used. Here, VLAN information is tagged to the standard Ethernet frame. The packets on the ISL link contain a standard Ethernet, FDDI, or Token Ring frame and the VLAN information associated with that frame.

\section{Basic Design of a Wi-Fi Network for Shared Internet}

It is a pure Internet access, thinks that your browser is a program. There Marina for the reception of radio signals from the transmitting antenna. This may be standard antenna. Consequently Los Kerry has taken to save the bearing antenna. Now the operator or determined by the program of bridges and networks Internet connection. Failed wire, people, internet receiver antenna specific location, and then imagine that you have two different complex very close to each other, four, seven buildings were used to build a multi-level line in perspective.

\section{Motorola Canopy Antenna for Sharing Point to Multi Point}

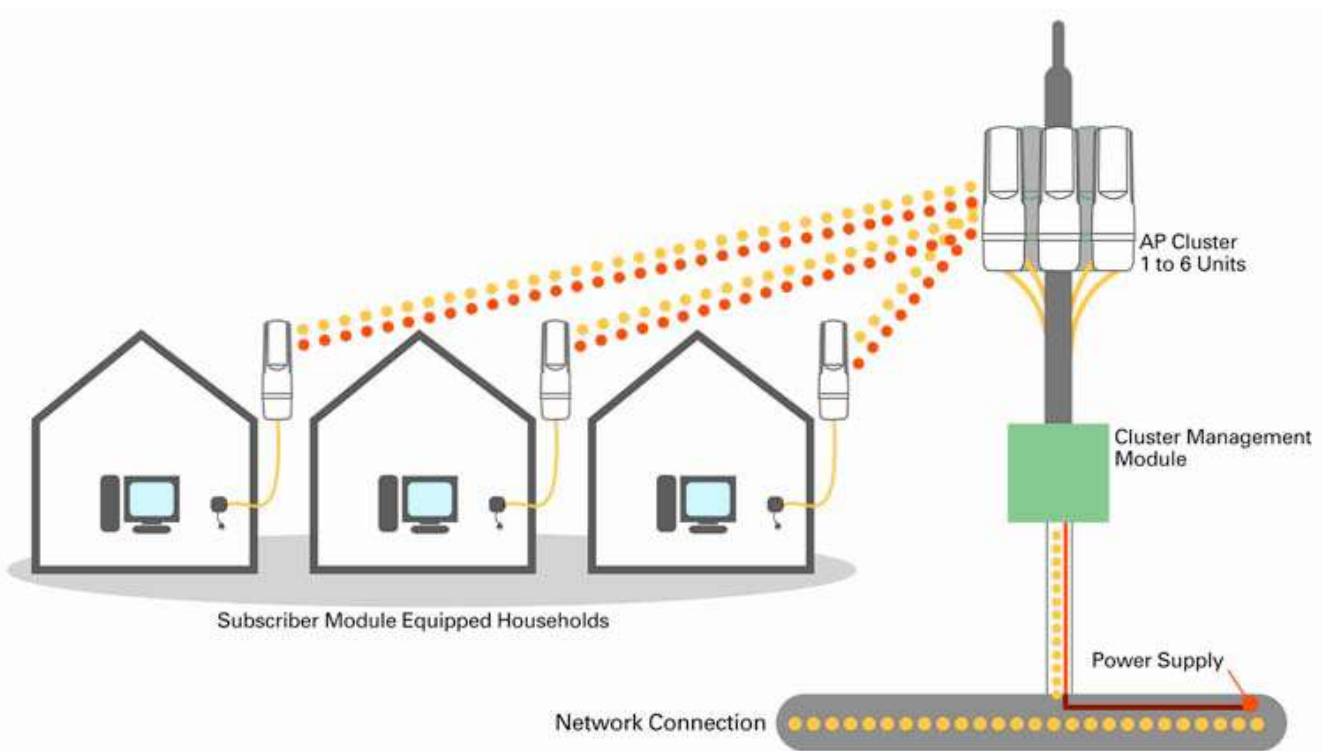

Figure 4. Point to Multipoint Sharing 


\section{Sharing of Internet Connection}

The main capacity (4 Mbps), can be divided into each stage of the other shades. Installation A1 B1 and the fields, and there was given to it is distributed to each of the number of Internet users of Nay, in all parts of the building. One cable CAT5 / 6 CAT antenna, with hardly and is infinite; the number of the connection (such as Motorola Canopy antenna) cannot be without the base stations. Each building has a common connection or other record can be made through the use of wireless access points. The second recording layer via a wireless connection $\mathrm{C} 1$ inside the building to connect to your ISP or settings for receiving antenna can work with.

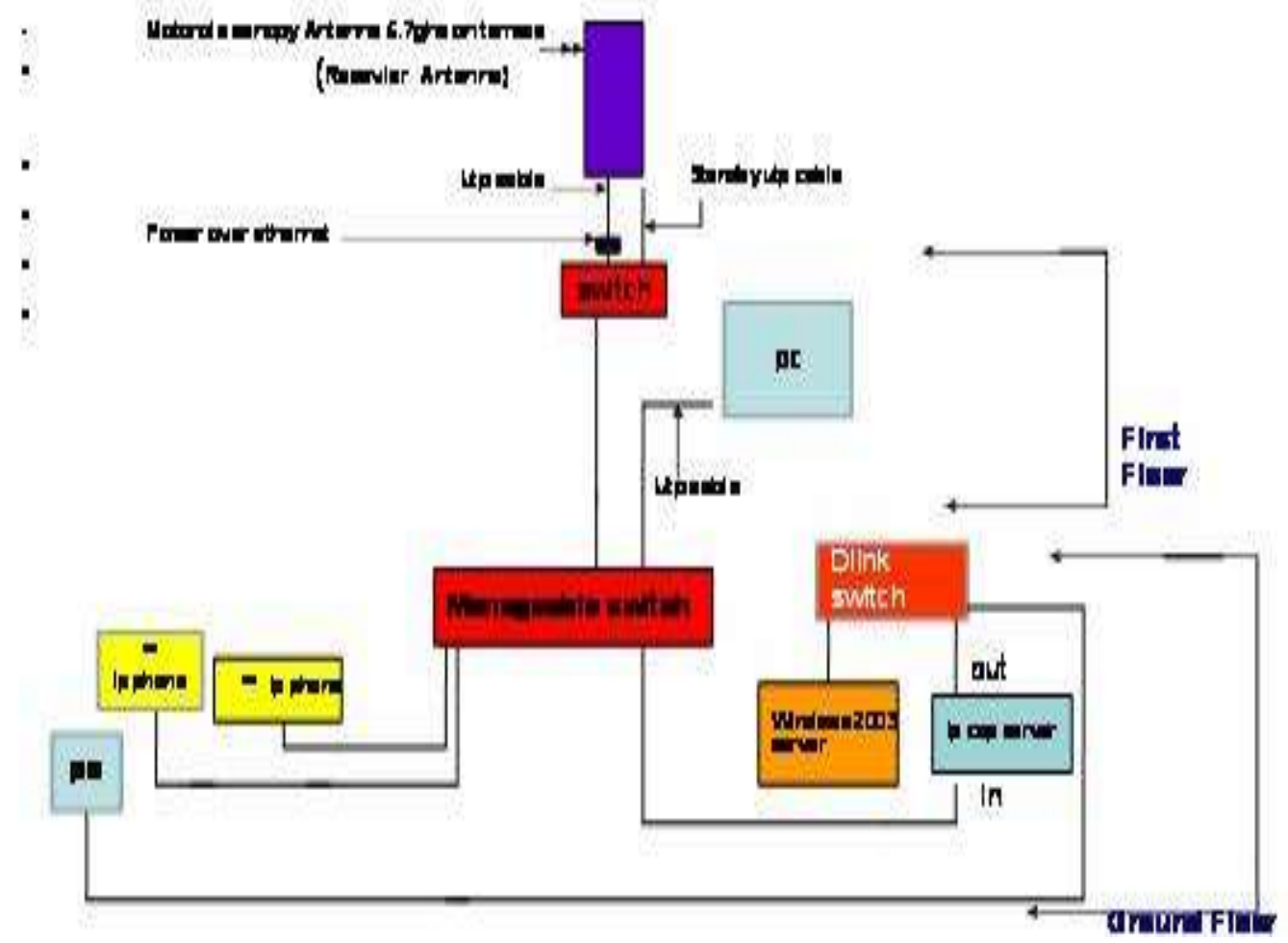

Figure 3. Location B1

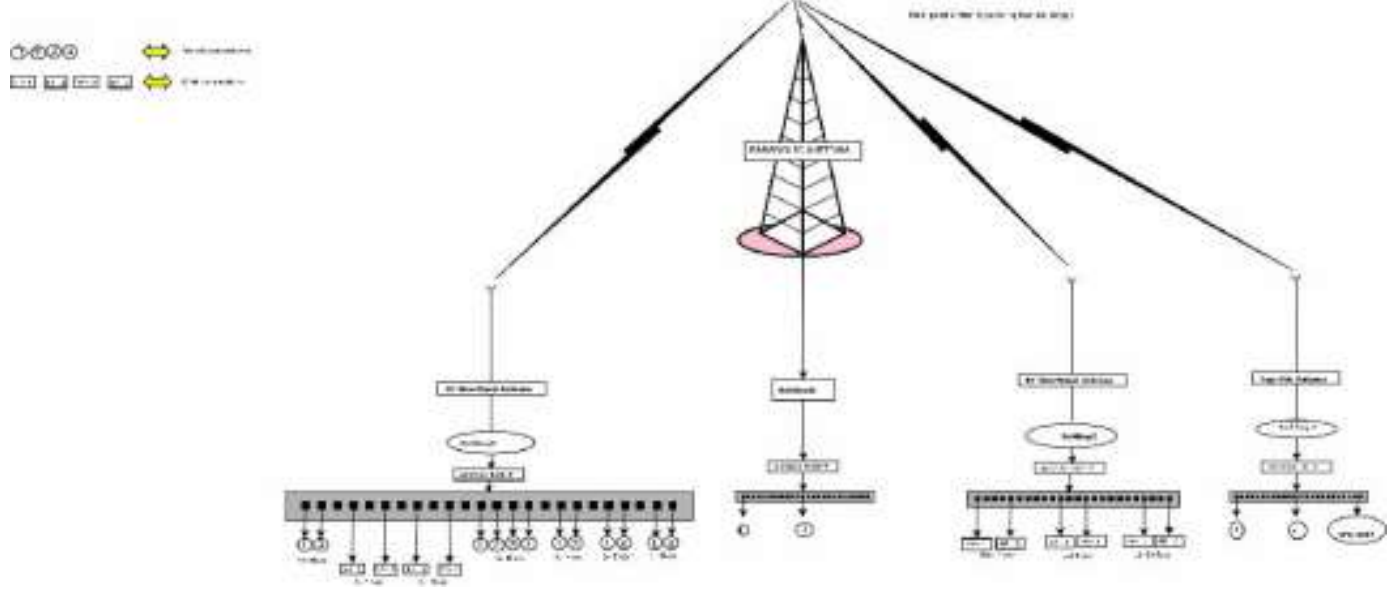

Figure 4. Location ID 


\subsection{Wi-Fi in MAN}

The Canopy system can also be used as an infrastructure for a metropolitan WiFi network. Canopy HotZone modules can be installed in urban areas to provide $2.4 \mathrm{GHz}$ public access to broadband services. In these applications, the Canopy system is used as an infrastructure to provide broadband access to the elements of the HotZone mesh network.

\section{Server Details of Network}

But we have to treat the servant of mourning for the side of the high-end customers. 3 servers, LAN Server, and it can be between us, there are three cards.

1) One server as Gateway which has Mikrotik router OS.

2) One server for content filtering

3) As a stand by

\subsection{Content Filtering Server}

Internet content filters can be very annoying. While trade through the school may want to prevent some unwanted websites, the law can be more difficult than expected. On the basis of need, anyone can create solutions with open source software, such as shrimp, squid and black list as guards. Squid server acts as an intermediary between the Web server and browser. To get the address of the browser application that connects to the content server as a proxy server, and then allows the browser to return. Content is also stored on the disc, and provide information to each other quickly in the near future to request the browser URL. This quickly leads to an efficient use of on-line and off. Is connected to a network that is a typical installation with two GTA proxy server, and is responsible for more Internet access (downloadable content) (hereafter hear Xubaarka HTTP request). Xubaarka standard can be found running on Red Hat Linux, simply install the RPM

\section{Conclusion}

In this article, sharing of Wi-Fi network is reviewed; in this article, citing the basic Wi-Fi design allows configuration, everyone with the network of basic knowledge. In addition, we have focused more on appearance and components. VLAN and VPN is also a comprehensive module for internet sharing over Wi-Fi. The configuration software will always be there to find the direction of the filter sites and more control of networks, etc.

\section{References}

[1] J. Schiller, "Mobile Communication", Pearson Education publications, Second Edition.

[2] E. Efstathiou and G. Polyzos, "A self-managed scheme for free citywide Wi-Fi”, World of Wireless Mobile and Multimedia Networks, 2005. WoWMoM 2005. Sixth IEEE International Symposium on a, (2005), pp. 502-506.

[3] Fon Wireless, Ltd. FON website. [Online] Available http://www.fon.com/, (2008).

[4] Freifunk Community. Freifunk. [Online] Available http://infrahip.hiit.fi/, (2008).

[5] T. Heer, "End-Host Authentication for HIP Middleboxes", Internet-Draft draft-heer-hip-midauth-00, Internet Engineering Task Force, Work in progress, (2007).

[6] T. Heer, S. Li and K. Wehrle, "PISA: P2P Wi-Fi Internet Sharing Architecture. P2P 2007", Seventh IEEE International Conference on Peer-to-Peer Computing, (2007), pp. 251- 252.

[7] InfraHIP Project. InfraHIP Project: HIPL. [Online] Available http://start.freifunk.net/, (2008).

[8] J. Jakobsen, Chillispot website. [Online] Available http://www.chillispot.info/, (2008).

[9] http://www.3com.com. 
[10] http://www.dlink.co.in.

[11] http://www.hyperlinktech.com/content/Tutorials.html.

[12] http://www.ipcop.org.

[13] http://www.linux.com/feature/60050.

[14] http://www.mikrotik.com.

[15] http://www.motorola.com/business/v/index.jsp?vgnextoid=20e284c606de6.110VgnVCM1000008406b0 OaRCR D

[16] http://www.petri.co.il.

[17] http://www.simulationexams.com/tutorials/ccna/vlan/ty pes-of-vlans.htm.

[18] http://www.simulationexams.com/tutorials/ccna/vlan/vlan communication.htm.

[19] http://www.simulationexams.com/tutorials/netplus/network-implementation/vlans.htm.

\section{Author}

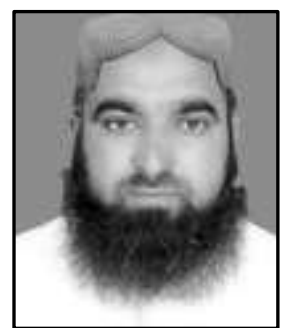

Javed Ahmed Shaheen, he is presently Studying Ph.D (CS) in from GC University Faisalabad Pakistan. 
International Journal of Future Generation Communication and Networking Vol. 10, No. 11 (2017) 